\title{
La notion de climat dans le discours de la presse anglophone : le traitement de la question climatique de 2010 à 2017
}

The notion of climate in the English-speaking press discourse: the coverage of climate change from 2010 to 2017

\section{Caroline Peynaud}

\section{OpenEdition \\ Journals}

Édition électronique

URL : https://journals.openedition.org/asp/5365

DOI : 10.4000/asp.5365

ISSN : 2108-6354

\section{Éditeur}

Groupe d'étude et de recherche en anglais de spécialité

\section{Édition imprimée}

Date de publication : 1 novembre 2018

Pagination : 77-93

ISSN : 1246-8185

Référence électronique

Caroline Peynaud, «La notion de climat dans le discours de la presse anglophone : le traitement de la question climatique de 2010 à 2017 », ASp [En ligne], 74 | 2018, mis en ligne le 01 novembre 2019 consulté le 21 septembre 2021. URL : http://journals.openedition.org/asp/5365 ; DOI : https://doi.org/ 10.4000/asp.5365

Ce document a été généré automatiquement le 21 septembre 2021.

Tous droits réservés 


\title{
La notion de climat dans le discours de la presse anglophone : le traitement de la question climatique de 2010 à 2017
}

\author{
The notion of climate in the English-speaking press discourse: the coverage of \\ climate change from 2010 to 2017
}

Caroline Peynaud

\section{Introduction}

1 Le climat et le changement climatique sont des questions complexes qui impliquent des connaissances issues de disciplines variées telles que la physique, la climatologie, la géographie, l'économie ou les sciences politiques (Colson et alii 2009). Il est pourtant essentiel que le grand public s'en saisisse et en comprenne les enjeux, et c'est en ce sens que la presse a un rôle à jouer dans la transmission de ces connaissances scientifiques sur le climat, à travers sa fonction de médiation (Moirand 2004:84; Merhy 2010:30 ; Beacco 1999 : 143). Si la médiation peut avoir plusieurs objectifs, celui de vulgariser la science peut être défini comme un processus de transmission de savoirs scientifiques et techniques dans le but de les rendre accessibles à des non-spécialistes (Beacco \& Moirand 1995 : 33 ; Charaudeau \& Maingueneau 2002 : 604). En ce sens, la presse répond à l'exigence de lisibilité, définie par P. Charaudeau (2005: 194) comme un «travail d'exposition le plus clair possible» qui a pour objectif de produire un discours compréhensible pour la majorité « à l'intérieur d'une cible préconstruite».

2 La manière dont la presse traite la question du climat influence d'abord l'évolution de la perception de cette question et l'état des connaissances du grand public sur ce sujet. Comme le souligne A. Carvalho : 
Public perception and attitudes with regard to [science and technology] are significantly influenced by representations of scientific knowledge conveyed by the press and other mass means of communication. (2007:223) notion de spécialisation telle qu'elle s'applique au domaine de la presse anglophone. Nous présentons ensuite le choix d'une étude en diachronie courte et le corpus qui a été constitué à cette fin.

\subsection{Presse et spécialisation dans le domaine du climat}

6 La presse est ici considérée comme une variété spécialisée de l'anglais, définie comme suit :

Since any specialized discipline or professional field is based on theory or practice that are expressed through language, language can serve as a starting point for further investigation into specific domains and specialized communities, and into their culture and discourse. (Resche op. cit. : 14)

ce cadre, culture et discours forment un continuum qui doit être analysé comme un tout, dans l'optique de comprendre comment une culture professionnelle donnée produit un discours qui répond à ses besoins. Le discours de la presse est marqué par de nombreuses contraintes qui s'exercent à deux niveaux. Tout d'abord, un ensemble de normes diffusées notamment par les associations professionnelles régissent la plupart des publications anglophone, par exemple, l'exigence de neutralité dans les articles informatifs, ou le respect de l'anonymat des sources, qui induisent un traitement particulier de l'actualité (Sloan \& Parcell 2002). Un second niveau de régulation intervient ensuite, celui de l'organe de presse publiant les articles, qui encadre la production du discours de plusieurs manières : vocabulaire à employer, longueur des articles, type de titres et ligne éditoriale, par exemple (Siegal 1999). Ces contraintes répondent aux objectifs de ce domaine spécialisé et conduisent à la production d'un discours caractérisé par le respect d'un ensemble de normes...

8 Le rôle de la presse, lorsqu'elle traite de sujets scientifiques, est de rendre ces sujets intelligibles pour le grand public (Moirand 1997). A. Bell (1994 : 263) a montré que les articles portant sur la science dans les médias contiennent une très faible proportion 
d'erreurs factuelles. Cependant, le traitement de la science par les médias reste complexe, dans la mesure où le fonctionnement de ces deux entités diffère à plusieurs niveaux. En effet, leur temporalité varie de vingt-quatre heures pour la presse quotidienne à plusieurs années pour la science; de plus, les journalistes sont à la recherche de faits alors que la science est faite d'incertitudes, d'hypothèses et de réfutations (ibidem : 260 ). Toutefois, la différence principale réside dans le fait que leurs objectifs ne sont pas les mêmes, dans la mesure où la presse produit un discours qui répond aux valeurs spécifiques de ce domaine spécialisé, notamment celles de proximité (ibid.: 264), d'équilibre des opinions, de nouveauté et de personnalisation (Boykoff \& Boykoff 2007 : 1191).

Depuis 2008 environ, de nombreuses recherches se sont intéressées à la représentation du changement climatique dans la presse, et notamment dans la presse écrite d'Europe et d'Amérique du Nord (Schäfer \& Schlichting 2014). L'analyse des discours de la presse a conduit plusieurs auteurs à remarquer que le traitement de la science est nécessairement teinté d'idéologie, influencée par la situation géographique dans laquelle les articles sont publiés, l'organe de presse concerné, ou simplement la vision dominante du monde à un moment donné (Carvalho 2007). Cette idéologie peut se traduire par un cadrage (framing [Entman 1993]) du débat qui s'appuie sur les titres, les chapeaux et les citations de sources, de manière différente selon l'identité propre de chaque journal (Dahl 2015). L'équilibre des opinions est également un biais, puisqu'il conduit à donner autant d'importance dans les articles à des opinions qui ne bénéficient pas du même crédit dans les communautés scientifiques (Boykoff \& Boykoff 2004). Ainsi, ceux qui nient l'origine humaine du réchauffement climatique sont aussi fréquemment représentés dans la presse que ceux qui affirment la responsabilité humaine, alors qu'ils sont largement minoritaires dans la communauté scientifique (ibid.). Pour S. Moirand, dans la presse, il ne s'agit pas de parler de science en tant que telle, mais de « construire des représentations du monde scientifique et de ses relations avec la politique et la société » $(2000: 2)$, et en accord avec les objectifs et les normes du discours de presse.

10 En conséquence, si la presse s'acquitte de son rôle de transmission de connaissances spécialisées, celle-ci a lieu dans le cadre très normé et fortement contraint du discours médiatique. Dans la mesure où le discours produit répond avant tout aux objectifs de la communauté professionnelle des journalistes de presse écrite, nous considérons que ce discours n'est pas spécialisé dans le domaine des sujets qui sont traités, ici, l'environnement, mais dans celui de la presse. Cette remarque s'applique de la même manière au journalisme scientifique qui, s'il s'agit bien de journalisme spécialisé en science, répond tout autant aux normes et aux objectifs de la presse, et non à ceux de la science. Dès lors, nous nous posons la question de savoir comment la presse traite la question complexe du climat en répondant aux besoins spécifiques du domaine spécialisé.

\subsection{Analyse de corpus en diachronie courte}

11 Cette étude s'appuie sur la combinaison d'une approche quantitative de linguistique de corpus, fondée sur des données statistiques, et d'une analyse de discours en contexte, afin de répondre à la double exigence de représentativité et de contextualisation formulée par M. Handford pour l'analyse des discours spécialisés : 
Corpora have much to say about language, but they can be lacking in contextual interpretability; genres are intrinsically contextual entities, but their linguistic features may be under-exposed. (2010:255)

12 Ce type d'analyse est possible concernant les discours spécialisés étant donné la taille plus réduite des corpus collectés pour ce type d'étude (ibid:258). Comme le remarque A. Koester :

Where very large corpora, through their de-contextualisation, give insights into lexico-grammatical patterns in the language as a whole, smaller specialised corpora give insights into patterns of language use in particular settings. (2010:69)

13 Cette analyse, qui porte sur les années 2010 et 2017, se place dans une optique de diachronie courte. Cette approche a acquis une légitimité dans l'étude du lexique général et spécialisé (Siouffi et alii. 2012) et se révèle particulièrement pertinente dans des domaines récents qui évoluent rapidement (Dury \& Picton 2009), tel celui de l'environnement. Elle permet notamment de mettre en lumière des dynamiques entourant l'émergence d'un fait nouveau (Siouffi et alii, op. cit.) et de cerner des phénomènes d'innovation linguistique.

14 Le corpus composé pour cette étude est donc un corpus de taille réduite, mais qui prend en compte l'ensemble des articles publiés sur le climat dans les journaux retenus, pour respecter le critère de représentativité. Il sera analysé de manière automatique, et également de manière qualitative, afin de faire émerger les phénomènes de variation dans une période de temps courte et récente.

\subsection{Corpus}

15 Le corpus constitué pour cette étude fait partie d'un corpus plus large réuni autour du thème de la justice climatique par une équipe interne du GREMUTS, à l'université Grenoble Alpes, et qui s'étend de 2014 à 2017. Les articles de 2017 ont été extraits de ce grand corpus, tandis que ceux de 2010 ont été ajoutés au corpus existant pour les besoins de cette étude. Le choix d'une étude diachronique portant sur 2010 a nécessairement restreint le nombre de journaux pris en compte dans le corpus de référence, pour des raisons de disponibilité des sources. Le corpus constitué selon les principes énoncés dans la section précédente a été tiré de deux journaux quotidiens, le New York Times, de tendance démocrate, et le Daily Telegraph, journal conservateur, qui représentent en outre deux aires géographiques distinctes. Il contient 329646 mots ; le tableau 1 décrit la répartition des mots au sein des quatre sous-corpus.

Tableau 1. Nombre de mots par sous-corpus

\begin{tabular}{|l|l|l|}
\hline & New York Times & Daily Telegraph \\
\hline 2010 & 91635 & 66589 \\
\hline 2017 & 140863 & 30559 \\
\hline
\end{tabular}

16 La règle de composition du corpus a été la mention du mot climate dans un contexte atmosphérique, c'est-à-dire que les articles qui contenaient ce mot pour évoquer le climat politique, par exemple, n'ont pas été retenus. Le nombre d'articles dans chaque journal et pour chaque période dépend du nombre d'articles publiés sur le sujet, ce qui 
explique le déséquilibre entre les sous-corpus. Cependant, il aurait semblé artificiel de supprimer des articles dans le corpus New York Times pour réduire ce déséquilibre, nous avons donc choisi de conserver le déséquilibre et de pondérer les résultats en fonction du nombre de mots dans chaque sous-corpus pour effectuer des comparaisons. Nous avons pris en compte l'ensemble des genres publiés sur ce sujet afin de ne pas exclure certains articles qui pouvaient être pertinents, dans la mesure où nous considérons que le discours journalistique est un tout cohérent, publié par des organes de presse qui exercent un fort contrôle sur le discours produit, ce qui devrait produire un lissage du produit final. Si les distinctions de genre sont certainement pertinentes, dans des contextes où la différence entre opinion et information est centrale, par exemple, nous avons privilégié dans ce travail la cohérence des articles dans chaque journal et l'analyse des différences entre aires géographiques et années de publication.

17 La recherche principale qui a été menée sur ce corpus a porté sur le mot climate, dont 2096 occurrences ont été relevées en tout. Il a été analysé à l'aide du logiciel de textométrie TXM (Heiden et alii. 2010) et, plus particulièrement, avec son outil de recherche des cooccurrents et son outil intégré d'étiquetage des parties du discours (TreeTagger). La même méthode a été appliquée aux quatre sous-corpus et nous avons utilisé la variable de l'indice de coocurrence (entre 2 et 4 ) afin d'adapter les résultats à la taille de chaque sous-corpus. L'indice de coocurrence est défini comme "un indicateur de probabilité de rencontre " entre deux mots dans un corpus (ibid.: 89). Plus l'indice est élevé, plus la coocurrence de deux mots est significative dans le corpus.

Afin de pouvoir interpréter précisément les résultats ainsi obtenus, il est nécessaire de prendre en compte le contexte global des événements liés au climat qui ont eu lieu lors de la période concernée. Dans cette brève chronologie, les événements liés à la politique des États-Unis sont dominants, dans la mesure où ils sont mentionnés dans les deux journaux, tandis que l'on trouve peu d'articles qui portent sur la politique britannique, y compris dans les articles du Daily Telegraph.

En 2009, le scandale du Climategate émerge. Il concerne des fuites de messages internes de chercheurs du Climatic Research Unit de l'université d'East Anglia, l'une des sources d'information du GIEC (Groupe d'experts intergouvernemental sur l'évolution du climat). Ces messages semblent accuser le GIEC de manipulation de résultats et de dérives déontologiques et remettent en cause de manière durable sa légitimité. La même année, la COP15 se tient à Copenhague. Elle est généralement considérée comme un échec dans la mesure où elle n'a pas abouti à un accord contraignant portant sur des objectifs quantitatifs de réduction des gaz à effet de serre.

20 La conférence de Cancùn, COP16, intervient en 2010 dans ce contexte difficile. Les COP suivantes font l'objet de peu de couverture dans la presse, jusqu'à la COP21, qui a lieu fin 2015 à Paris, et qui aboutit aux accord de Paris, souvent mentionnés dans le corpus.

21 Un an plus tard, Donald Trump est élu à la présidence des États-Unis. Durant la campagne présidentielle, il affiche clairement son scepticisme envers le fait que les activités humaines seraient à l'origine du phénomène de réchauffement climatique. Cette position évolue quelque peu au cours de son mandat mais, en juin 2017, le président prend néanmoins la décision de retirer les États-Unis de l'accord de Paris. Parallèlement, de nombreux élus américains, y compris des membres du parti républicain, s'engagent en faveur du combat contre le changement climatique et contre les positions prises par Donald Trump. 
fois ce corpus constitué et replacé dans son contexte historique, nous avons cherché à comparer les contextes dans lesquels le mot climate est employé dans les différents sous-corpus.

\section{Approche quantitative : les cooccurrents de climate}

Nous avons tout d'abord cherché à identifier les coocurrents les plus courants de climate dans le corpus. Ces analyses ont été réalisées en trois temps. Tout d'abord, nous avons orienté la recherche sur le contexte droit afin de dresser une liste des mots apparaissant immédiatement après climate (cooccurrents +1$)$. Nous avons ensuite cherché dans le contexte gauche les mots qui sont employés un ou deux mots avant climate (cooccurrents - 2). Pour chaque requête, nous avons analysé les résultats dans le corpus global, puis dans chaque sous-corpus, afin de les comparer entre eux. Nous avons enfin cherché à vérifier nos résultats dans le contexte plus large des articles, à l'aide du calcul de spécificités pour chacun des corpus.

\subsection{Le contexte droit (cooccurrents +1 )}

24 Un premier repérage des cooccurrents fait apparaître la forte prédominance de l'usage de climate dans l'expression climate change. Change est en effet le cooccurrent principal de climate, avec lequel il se trouve en co-fréquence dans $89 \%$ des occurrences en 2010 et dans $69 \%$ en 2017. On peut en déduire que la question du climat est le plus fréquemment abordée sous l'angle du changement climatique dans les articles des quatre sous-corpus. Parmi les autres cooccurrents du contexte droit, nous avons relevé trois catégories sémantiques principales : les références à la science, aux négociations internationales et aux attitudes vis-à-vis du climat.

Le champ sémantique de la science, représenté par science (61ํ), scientists (32), research (8) ou models (7), est le plus fréquent dans le corpus global, avec des indices très élevés pour science et scientists, ce qui met en lumière le fait que le climat est présenté comme une question scientifique avant tout, ce qui accroît la légitimité des connaissances produites à ce sujet, comme dans l'exemple suivant :

One government scientist who worked on the report, Katharine Hayhoe, a professor of political science at Texas Tech University, called the conclusions among "the most comprehensive climate science reports" to be published. (NYT172)

L'analyse des lignes de concordance met néanmoins en évidence le fait que la science est souvent convoquée pour être remise en question, dans le cas de références aux climato-sceptiques: "Republicans, who control the White House and Congress, remain broadly skeptical of climate science and rely heavily on the electoral support of oil- and coalproducing states» (NYT17). Dans tous les cas, ces deux exemples et l'analyse plus large des emplois de ce champ lexical font apparaître que la science du climat est traitée comme un objet bien défini composé de faits établis que l'on peut affirmer ou contester, et non comme une recherche continue faite de nombreuses incertitudes, ce qui correspondrait davantage à la manière dont cette question est perçue par les scientifiques.

De plus, des disparités apparaissent entre les sous-corpus. En premier lieu, les références à la science sont beaucoup plus fréquentes dans le New York Times que dans le Daily Telegraph. Dans NYT17, scientists a un indice de 26, et science un indice de 34, ce 
qui met en évidence leur fréquente cooccurrence avec climate. Dans le Daily Telegraph, la co-fréquence est nettement moins significative puisque l'indice de science est de 4 pour 2010, et qu'aucun mot faisant référence à la science n'a un indice de plus de 2 en 2017. Il semble que les références au climat soient étroitement liées à la science dans le New York Times, alors que ce n'est pas le cas dans le Daily Telegraph.

Les références aux politiques climatiques ou aux négociations sur le climat sont très fréquentes également, de manière homogène dans les quatre sous-corpus, notamment avec de nombreuses références aux accords de Paris en 2017. Celles-ci sont principalement liées à la décision du président des États-Unis de se retirer de ces accords. Les références aux différentes étapes des négociations sur le climat sont tout à fait en adéquation avec la valeur de proximité à laquelle les journalistes adhèrent, dans la mesure où il s'agit d'un événement clairement identifiable, qui est facile à situer dans le temps et qui se déroule au moment où la presse en parle. Cette caractéristique peut expliquer pourquoi les COP (Conferences of the Parties) bénéficient d'une large couverture médiatique, alors que les avancées scientifiques sur le climat, dont la temporalité est moins en adéquation avec celle de la presse, sont plus difficiles à traiter.

Enfin, nous trouvons en coocurrence avec climate de nombreuses références aux attitudes envers le changement climatique, parmi lesquelles nous identifions trois attitudes principales : le déni, le choc devant les risques liés au climat, et les actions à mener. Dans les quatre sous-corpus, nous relevons des mots tels que skeptics (18) ou debate (4), des références à ceux qui doutent du changement climatique ou de son origine humaine, ce qui met en évidence le fait que la presse respecte la valeur d'équilibre, par laquelle ils donnent voix à toutes les opinions. Nous remarquons également une évolution dans la composition de cette catégorie dans les deux journaux, entre 2010 et 2017. En 2010, les coocurrents les plus fréquents dans les deux journaux font référence aux opinions opposées sur le climat (skeptics [16]), camp [4]), alors qu'en 2017, ce sont les références aux actions à mener qui dominent (combat [5], action [4], adaptation [6], tackling [7], address [3]), par exemple :

People in every sector from national governments, cities, states, youth movements, faith groups and businesses, became more determined than ever to continue the fight for a global programme of climate action that would protect economic growth, create jobs, improve air quality and public health and secure a stable climate for generations to come. (DT17)

De manière générale, si les références à la science sont dominantes dans les deux souscorpus du New York Times, c'est cette dernière catégorie, qui concerne les actions à mener, qui est la plus fréquente dans les deux sous-corpus du Daily Telegraph.

\subsection{Le contexte gauche (coocurrents -2)}

31 La recherche des coocurrents du contexte gauche permet de faire apparaitre, notamment, les adjectifs et les verbes qui précèdent climate. Cette mesure révèle, dans un premier temps, que la plupart des verbes employés avant climate font référence aux actions qui peuvent être menées pour le climat, ou contre le changement climatique. Les quatre sous-corpus emploient le même type de vocabulaire : address (15), fight (17), tackle (12) et combat (9) sont les verbes les plus fréquents dans le corpus global. Le corpus DT10 se distingue cependant par l'emploi des mots avert (4), avoid (3) et prevent (2), qui sont absents des autres corpus. Sans doute les évolutions de la science ont-elles 
rendu moins pertinente l'hypothèse d'un évitement du changement climatique en 2017, mais il est alors surprenant que ces mots n'apparaissent pas du tout dans NYT10.

La deuxième catégorie est celle des conséquences du changement climatique, avec des mots tels que dangerous (5), effects (16), risks (12) ou impact (14). Ce champ lexical est employé pour insister sur l'importance de la question climatique, comme c'est le cas dans l'exemple suivant :

Among the more significant of the study's findings is that it is possible to attribute some extreme weather to climate change. The field known as "attribution science" has advanced rapidly in response to increasing risks from climate change. (NYT17)

Si ce champ lexical apparaît dans la plupart des sous-corpus, il est presque absent de DT10. Dans les deux sous-corpus de 2010, nous trouvons cependant les adjectifs chief (6), top (5) et prominent (3) qui ne sont pas présents dans le reste du corpus. Nous suggérons que l'effet rhétorique de l'emploi de ces adjectifs est similaire à celui de la description des conséquences, c'est-à-dire qu'ils ont pour objectif de convaincre de l'importance de la question climatique. Le fait que ce type d'adjectifs n'apparaît qu'en 2010 signale sans doute qu'en 2017, mentionner l'importance de la question a un effet de persuasion moindre, dans la mesure où le problème du climat est bien connu. Les références aux conséquences plus précises du changement climatique ont sans doute alors plus d'impact sur les lecteurs. Nous trouvons d'ailleurs en 2017 de nombreuses références à business risks, financial risks ou flood risk, par exemple, qui donnent une image plus spécifique des conséquences possibles du changement climatique, ce qui est sans doute considéré comme plus pertinent pour les lecteurs de 2017.

\subsection{Spécificités de chaque corpus et mise en contexte}

Afin d'obtenir une représentation plus juste du contexte dans lequel ces cooccurrents apparaissent, nous avons conduit une recherche de spécificités dans l'ensemble du corpus. L'outil spécificités de TXM permet de mettre en évidence les spécificités de chaque sous-corpus par rapport au corpus global. Par exemple, pour chaque période, cet outil met en lumière les événements, les personnes ou les lieux qui sont d'actualité : Trump, Pruitt, Paris ou Bonn en 2017, Copenhague ou Cancún en 2010.

Cette recherche montre que les références aux solutions contre le changement climatique sont l'une des spécificités des deux sous-corpus du Daily Telegraph avec green (13), energy (11), ou low-carbon (9) en 2010, et energy (27), renewable (16), green (13) ou solar (10) en 2017. La question des énergies renouvelables est traitée de manière fréquente dans ce journal. Or, ce champ lexical n'apparaît pas dans les résultats de l'analyse du New York Times, ce qui ne veut pas dire qu'il est absent du corpus, mais simplement que sa fréquence n'en fait pas une spécificité de ce sous-corpus. Dans le New York Times, ce sont les références aux effets du changement climatique qui dominent, avec ice (18) ou warming (18) en 2010, et health (9) ou hurricanes (14) en 2017. Nous avons également déjà mentionné les références à la science, particulièrement présentes dans les deux sous-corpus de ce journal.

Cette dernière analyse permet de mettre en perspective les résultats sur les cooccurrents. La concentration sur les effets observables du changement climatique dans le New York Times est cohérente avec les appels à la science remarqués dans la section précédente au sens où ces deux procédés révèlent une volonté de convaincre de la réalité ou de la nécessité d'agir, en 2010 comme en 2017. Le Daily Telegraph, en 
revanche, se concentre davantage sur les actions à mener, dont les énergies renouvelables font partie. Dans l'optique d'affiner cette analyse, nous avons conduit une recherche des sources d'informations sur le climat.

\section{Les sources d'information}

Le repérage des sources des journalistes sur le climat permet d'identifier ceux qui sont considérés comme des experts de cette question dans la presse. À l'aide de l'outil d'étiquetage des parties du discours intégré à TXM (TreeTagger), nous avons identifié les noms propres qui apparaissent en cooccurrence (10 mots avant, 10 mots après) avec climate. Ces experts ont été classés en trois catégories : les personnalités politiques, les scientifiques, et une catégorie «autres " qui contient principalement des artistes dans le Daily Telegraph, comme le peintre Eduardo Gold, et des journalistes ou des membres d'ONG pour le New York Times.

La figure 1 montre la répartition de ces catégories dans les quatre sous-corpus, en fonction du nombre de personnes différentes citées dans chaque sous-corpus. Si les nombres sont relativement faibles, c'est avant tout parce que cette recherche ne porte pas sur l'ensemble des sources citées, mais sur celles qui apparaissent en cooccurrence avec climate avec un indice de 2, ce qui opère une première sélection. La figure 1 présente uniquement les personnes les plus significatives, celles qui sont citées plusieurs fois dans le corpus.

Figure 1. Répartition des sources par catégorie

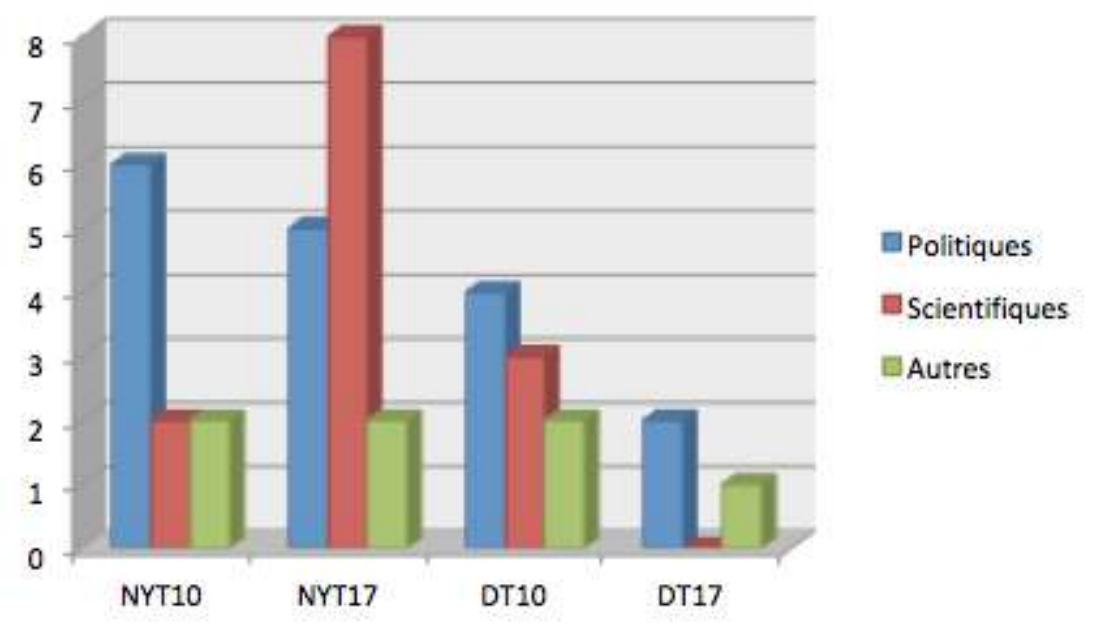

De manière générale, la politique des États-Unis est mentionnée dans les quatre souscorpus, alors que la politique européenne est presque absente du New York Times (avec une référence à Angela Merkel seulement). Partout, les politiques sont soit des dirigeants (Scott Pruitt, Donald Trump, John Kerry, Barack Obama, Gordon Brown), soit des spécialistes du climat; ces derniers peuvent alors être considérés comme des experts. C'est le cas de Chris Huhne, "energy and climate change secretary" (42 occurrences dans 18 articles), ou Xie Zhenhua, "the top Chinese climate official » (4 occurrences dans 4 articles). Ceux-ci sont cités comme des sources légitimes d'information concernant les politiques climatiques, mais rarement comme sources à propos de la science du climat, comme l'illustre l'exemple suivant : 
However, Chris Huhne, the Energy and Climate Change Secretary, has said Britain would continue the policy of the last administration by committing to cut emissions first before asking poorer countries to take action.

de la presse des traces de l'évolution politique des pays concernés. Les controverses actuelles sur le climat aux États-Unis se reflètent dans les sources convoquées dans le New York Times, qui cite un nombre accru de scientifiques issus de disciplines variées. Ce procédé accroît la légitimité du discours par un processus d'« effacement énonciatif » (Adam \& Lugrin 2006) par lequel le journaliste efface sa propre voix pour laisser la parole aux personnes citées, considérées légitimes dans le domaine concerné. Les scientifiques sont appelés à convaincre de la réalité du changement climatique en faisant appel à des disciplines diverses, qui montrent quels domaines pourraient être affectés par ce phénomène. L'information sur le climat dans NYT10 semble au contraire se concentrer sur la climatologie, à l'exclusion d'autres disciplines, ce qui suggère une information concentrée sur le climat en lui-même moins que sur ses effets. Dans le Daily Telegraph, l'évolution est inverse. En 2010, comme pour le New York Times, des climatologues sont appelés à informer le public à propos du changement climatique, par exemple : «Dr Peter Stott, head of climate monitoring at the Met office, said there was 
'clear evidence' of a rise in the frequency of extreme weather events due to climate change ". Cependant, en 2017, plus aucun scientifique n'apparaît dans la liste de fréquence, ce qui signale que, s'ils sont cités dans les articles, ce n'est que de manière ponctuelle. Ce constat est cohérent avec l'idée, qui était suggérée par l'analyse des cooccurrents, que le discours du Daily Telegraph en 2017 ne reflète plus le même besoin de convaincre, par le recours à la science, que le changement climatique est réel.

\section{Approche qualitative du contexte immédiat de « climate change »}

44 Nous avons enfin mené une recherche plus qualitative fondée sur le repérage des occurrences de climate change. Comme il a été mentionné plus tôt, la majorité des occurrences de climate se trouvent en cooccurrence avec change, ce qui explique pourquoi nous avons choisi de nous concentrer sur ce nom composé. Le contexte immédiat de cette expression, gauche et droit, a été analysé afin de vérifier dans le détail les résultats obtenus automatiquement et de tenter de saisir ces textes de manière plus précise. Nous nous concentrons ici sur l'évolution diachronique entre 2010 et 2017.

Nous avons remarqué dans les sections précédentes que l'un des champs sémantiques les plus fréquents parmi les cooccurrents était celui du combat contre le changement climatique, qui apparait dans les quatre sous-corpus. La lecture du contexte immédiat révèle que ces références évoluent entre 2010 et 2017. En 2010, nous trouvons de nombreuses occurrences de avoid climate change ou de adapt to climate change. L'idée de s'adapter au changement climatique est centrale dans le troisième rapport du GIEC, publié en 2001. Or, en 2010, cette idée est toujours très fréquemment mentionnée dans la presse : "Whatever the cost is of avoiding climate change - and we might think it is high it's much lower than the costs of not avoiding dangerous climate change,' he said " (DT10). Des expressions telles que "dangerous climate change » sont d'ailleurs tout à fait absentes du corpus de 2017, démontrant sans doute que la dangerosité de ce phénomène n'a plus besoin d'être affirmée, qu'elle est connue du grand public à cette période.

En 2017, l'idée d'éviter le changement climatique ou de s'y adapter n'est plus centrale. Le cinquième rapport du GIEC, publié en 2014, se concentre davantage sur les aspects socio-économiques du changement climatique et leurs conséquences pour le développement durable (GIEC 2014). Dès lors, les verbes employés sont bien plus généraux, tels que tackle ou combat, ou encore : "democracies have vowed to pursue efforts to curb climate change despite the rift caused by its withdrawal from the Paris Agreement " (DT17). Nous voyons ici comment les évolutions scientifiques au sein du domaine influencent la manière dont la presse représente ce domaine pour le grand public.

L'analyse des cooccurrents a également révélé des références fréquentes à ceux qui remettent en cause l'origine humaine du changement climatique. Là encore, l'analyse du contexte peut permettre de préciser les différents groupes dont il est question. En 2010, ceux qui ne croient pas au changement climatique font partie de groupes variés, qui ne sont pas toujours définis précisément, par exemple :

After all, national surveys released during the last eight months have been interpreted as showing that fewer and fewer Americans believe that climate change is real, human-caused and threatening to people. (NYT10) 

notion de changement climatique en 2010, comme le soulignent plusieurs références à l'UEA (University of East Anglia) : " 'When the UEA emails were leaked it cast a huge cloud of doubt over the whole issue of climate change,' he said» (DT10). La situation est bien différente en 2017, où l'ensemble des références aux deniers, ceux qui nient l'origine humaine du changement climatique, ont un lien avec le président Trump et son gouvernement : "We have a president who dismisses climate change as a hoax» (NYT51). Ainsi, s'il est vrai que la presse donne voix à toutes les opinions, y compris à celles qui sont scientifiquement minoritaires, elle présente également les changements au sein de ces groupes minoritaires et reflète ainsi l'évolution globale de l'opinion sur cette question.

49 Ce doute plus général en 2010 est sans doute la raison pour laquelle le sous-corpus de 2010 est le seul dans lequel nous trouvons des contextes de climate change qui font référence à la nécessité de prouver le changement climatique :

STANDING on the deck of this floating laboratory for Arctic science, which is part of Canada's Coast Guard fleet and one of the world's most powerful icebreakers, I can see vivid evidence of climate change. (NYT10)

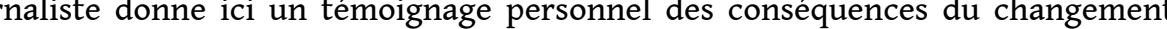
climatique dans lequel il cherche à décrire et à convaincre le grand public de la réalité de ses effets. Cette volonté d'informer est souvent exprimée explicitement :

Seeing danger in the divide between climate scientists and meteorologists, a variety of groups concerned with educating the public on climate change - including the National Environmental Education Foundation, a federally financed nonprofit, and Yale - are working to close the gap with research and educational forums. (NYT10)

Ce type de témoignage n'est plus relayé par la presse en 2017. En revanche, la spécificité des contextes de 2017 est le fait que le lien entre la question du climat et l'économie ou la politique est très fréquemment mis en avant :

The conference reviewed studies and data that have emerged since Francis issued a forceful 2015 encyclical that highlighted the social and ethical issues caused by climate change, including mass migration and famine. (NYT17)

The G.A.O. study draws on interviews with 26 scientific and economic experts and 30 studies, though it focuses most heavily on the only two national-scale studies analyzing the economic effects of climate change. (NYT17)

Il ne s'agit plus seulement de décrire des problèmes lointains, comme la fonte des glaces dans l'Arctique, mais de décrire précisément les conséquences économiques, politiques et sociales du changement climatique ; la question est davantage ancrée dans les enjeux sociétaux actuels. L'évolution dans le temps du traitement de cette question apparaît clairement dans cette analyse.

\section{Conclusion}

53 À travers cette étude, nous avons souhaité montrer que l'analyse de corpus peut bénéficier d'une combinaison d'approches quantitatives et d'approches qualitatives, où les premières permettent de poser des hypothèses que les secondes confirment, réfutent ou précisent. Grâce à cette méthodologie, nous avons pu dégager des tendances à la fois dans l'évolution du traitement du climat entre 2010 et 2017 et entre les aires géographiques. 

change ". Nos analyses ont révélé que cette affirmation devait être nuancée. Si les deux journaux soulignent bien la nécessité de prouver le changement climatique en 2010, notamment par les appels fréquents à la science, leur évolution n'est pas parallèle. En 2017, le débat semble en effet clos pour le Daily Telegraph, qui se concentre sur les actions concrètes à mettre en place, à travers les énergies renouvelables, par exemple. Dans le New York Times, en revanche, il y a toujours une claire recherche de persuasion, notamment à travers l'appel à un nombre croissant de scientifiques, qui peut être interprété comme une prise de distance face aux décisions politiques récentes de l'Administration Trump. Une étude plus large incluant davantage de journaux dans chaque pays permettrait de neutraliser le biais éventuel de la ligne éditoriale des journaux.

ette étude montre clairement l'apport de la diachronie courte dans un domaine tel que celui du climat, en constante évolution et dans lequel l'information du public est une question cruciale. Même sur une période réduite de sept ans, les différences de traitement sont sensibles et il est possible d'observer des évolutions très claires.

Enfin, si plusieurs variations ont pu être soulignées, il demeure un élément de stabilité dans l'ensemble du corpus : celui du discours de la presse. Nous avons pu déterminer que la valeur d'équilibre de la représentation des opinions opposées est respectée dans l'ensemble du corpus et que les articles se concentrent sur les événements récents, tels que les conférences ou les décisions politiques, plus que sur la représentation des avancées scientifiques. Les références à la science restent d'ailleurs très générales puisqu'aucun terme technique n'apparait dans le traitement automatique du corpus, ce qui est cohérent avec la nécessité de produire un discours accessible au plus grand nombre. Cette étude a ainsi permis de mettre en évidence la manière dont le domaine technique du climat est intégré dans le discours normé de la presse écrite.

L'auteur tient à remercier le consortium CORLI du CNRS et le Grenoble Alpes Data Institute (projet IDEX) qui ont fourni les financements grâce auxquels ce corpus a pu être constitué.

\section{BIBLIOGRAPHIE}

Adam, Jean-Michel \& Gilles Lugrin. 2006. «Effacement énonciatif et diffraction co-textuelle de la prise en charge des énoncés dans les hyperstructures journalistiques ». Semen. Revue de sémiolinguistique des textes et discours 22 «Énonciation et responsabilité dans les médias ». Consulté le 10/7/2018 <http://semen.revues.org/document4381.html>.

Beacco, Jean-Claude. 1999. L'astronomie dans les médias : analyses linguistiques de discours de vulgarisation. Paris : Presses de la Sorbonne Nouvelle.

Beacco, Jean-Claude \& Sophie Moirand. 1995. « Autour des discours de transmission des connaissances ». Langages 29/117, 32-53. Consulté le 10/7/2018 <https://doi.org/10.3406/lgge. 1995.1704>. 
Bell, Allan. 1994. « Media (mis)communication on the science of climate change ». Public Understanding of Science, 259-275.

Boykoff, Maxwell T. \& Jules M. Boykoff. 2004. «Balance as bias: Global warming and the US prestige press ». Global Environmental Change 14/2, 125-36. Consulté le 10/7/2018 <https:// doi.org/10.1016/j.gloenvcha.2003.10.001>.

Boykoff, Maxwell T. \& Jules M. Boykoff. 2007. « Climate change and journalistic norms: A casestudy of US mass-media coverage ». Geoforum 38/6, 1190-1204. Consulté le 10/7/2018 < doi.org/ 10.1016/j.geoforum.2007.01.008>.

Carvalho, Anabela. 2007. «Ideological cultures and media discourses on scientific knowledge: rereading news on climate change $»$. Public understanding of science 16/2, 223-243.

Charaudeau, Patrick \& Dominique Maingueneau. 2002. Dictionnaire d'analyse du discours. Paris : Seuil.

Colson, Renaud, Encinas de Munagorri, Benjamin Denis, Olivier Leclerc \& Sandrine Rousseaux. 2009. Expertise et gouvernance du changement climatique. LGDJ : Paris.

Dahl, Trine. 2015. « Contested science in the media: Linguistic traces of news writers' framing activity ». Written Communication 32/1, 39-65. Consulté le 10/7/2018 <https://doi.org/ 10.1177/0741088314557623>.

Dury, Pascaline \&Aurélie Picton. 2009. « Terminologie et diachronie: vers une réconciliation théorique et méthodologique?». Revue française de linguistique appliquée 14/2, 31-41.

Entman, Robert M. 1993. « Framing: Toward clarification of a fractured paradigm ». Journal of communication $43 / 4,51-58$.

Handford, Michael. 2010. « What can a corpus tell us about specialist genres ». In O’Keeffe, A. \& M. McCarthy (dir.), The Routledge Handbook of Corpus Linguistics. Londres : Routledge, 255-269. Heiden, Serge, Jean-Philippe Magué \& Bénédicte Pincemin. 2010. « TXM: Une plateforme logicielle open-source pour la textométrie-conception et développement ». In 10th International Conference on the Statistical Analysis of Textual Data-JADT 2010, 2, 1021-1032. Edizioni Universitarie di Lettere Economia Diritto.

Koester, Almut. 2010. « Building small specialised corpora ». In O’Keeffe, A. \& M. McCarthy (dir.), The Routledge Handbook of Corpus Linguistics. Londres : Routledge, 66-79.

Merhy, Layal. 2010. «La vulgarisation dans les médias : sciences et émotions ». Communication, lettres et sciences du langage 4/1, 29-41.

Moirand, Sophie. 1997. « Formes discursives de la diffusion des savoirs dans les médias ». Hermès 21. Consulté le 10/7/2018 <https://doi.org/10.4267/2042/15040>.

Moirand, Sophie. 2000. «Variations discursives dans deux situations contrastées de la presse ordinaire ». Carnets du CEDISCOR 6 « Rencontres discursives entre sciences et politiques dans les médias ", 45-62.

Moirand, Sophie. 2004. « De la médiation à la médiatisation des faits scientifiques et techniques : où en est l'analyse du discours? ». In Le Marec, J. \& I. Babou (dir.), Colloque Sciences, Médias et Société, 71-99.

Resche, Catherine. 2013. Economic Terms and beyond: Capitalising on the Wealth of Notions. Linguistic Insights. Berne : Peter Lang. 
Schäfer, Mike S. \& Inga Schlichting. 2014. « Media representations of climate change: A metaanalysis of the research field ». Environmental Communication $8 / 2 ; 142-60$. Consulté le 10/7/2018 <https://doi.org/10.1080/17524032.2014.914050>.

Siegal, Allan M. \& William G. Connolly. 1999. Manual of Style and Usage. New York : Random House.

Siouffi, Gilles, Agnès Steuckardt \& Chantal Wionet. 2012. « Comment enquêter sur les diachronies courtes et contemporaines?»SHS Web of Conferences 1, 215-26. Consulté le 10/7/2018 <https:// doi.org/10.1051/shsconf/20120100214>.

Sloan, David W. \& Lisa Mullikin Parcell (dir.). 2002. American Journalism, History, Principles, Practices. Londres : McFarland \& Company.

\section{NOTES}

1. Lorsque nous citons un cooccurrent, nous indiquons systématiquement l'indice de cooccurrence entre parenthèses.

2. Dans l'ensemble des analyses, nous faisons référence aux sous-corpus à l'aide des codes suivants : NYT17 (New York Times 2017), NYT10 (New York Times 2010), DT17 (Daily Telegraph 2017) et DT10 (Daily Telegraph 2010). Les articles sont identifiés en fonction des sous-corpus dont ils sont tirés.

\section{RÉSUMÉS}

La presse joue un rôle de médiation, de transmission des connaissances scientifiques pour le grand public. Ce rôle est essentiel dans un domaine comme celui du climat, dont les enjeux doivent être saisis par le plus grand nombre pour que des actions soient entreprises. Cette étude se propose d'analyser la manière dont la question du climat est traitée dans deux journaux anglophones, le New York Times et le Daily Telegraph, lors de deux périodes, 2010 et 2017. L'objectif est double : le traitement du climat dans la presse renseigne tout d'abord sur l'état de l'opinion à propos du climat à une période et dans une aire géographique données; il met également en évidence la manière dont la presse se saisit d'une question scientifique complexe pour l'intégrer dans le discours fortement normé qu'elle produit. Dans cette optique, des analyses statistiques de cooccurrences ont été conduites à l'aide du logiciel TXM, afin d'obtenir des résultats quantifiables, qui ont ensuite été mis en perspective par une étude plus qualitative des contextes de climate.

The press plays a mediation role, conveying scientific knowledge to the general public. That role is essential in a field like climate, where what is at stake needs to be understood by as many as possible so that appropriate action can be undertaken. The present study aims at analysing the way in which the issue of climate is treated in two newspapers, the New York Times and the Daily Telegraph, at two time periods, 2010 and 2017. The aim is twofold. First, the treatment of climate in the press provides an insight into public opinion on the matter at a given time and place. Secondly, it highlights the way in which the press appropriates such a complex scientific issue to 
embed it into the very normative discourse it produces. To understand these issues, statistical cooccurrence analyses have been performed using TXM software, to obtain quantifiable results. They were then put into a broader perspective using a more qualitative analysis of the contexts in which "climate" appears.

\section{INDEX}

Mots-clés : diachronie courte, discours du changement climatique, presse quotidienne, textométrie, variété spécialisé

Keywords : climate change discourse, daily press, short diachrony, specialised variety, textometry

\section{AUTEUR \\ CAROLINE PEYNAUD}

Caroline Peynaud est maître de conférences en anglais de spécialité à l'UFR de langues étrangères de l'université Grenoble Alpes, où elle enseigne principalement l'anglais économique et la grammaire ; elle est membre du laboratoire ILCEA4 - GREMUTS. Sa recherche porte sur le journalisme considéré comme domaine spécialisé, plus particulièrement sur la culture des journalistes aux États-Unis et sur les discours qu'ils produisent. Elle a publié dans ce domaine dans diverses revues (Anglophonia, ASp, ILCEA...). Caroline.peynaud@univ-grenoble-alpes.fr 